\title{
Sodium Chloride Enhances Recombinant Adeno-Associated Virus Production in a Serum-Free Suspension Manufacturing Platform Using the Herpes Simplex Virus System
}

\author{
Laura Adamson-Small, Mark Potter, Barry J. Byrne, and Nathalie Clément* \\ Department of Pediatrics, Powell Gene Therapy Center, University of Florida, Gainesville, FL.
}

The increase in effective treatments using recombinant adeno-associated viral (rAAV) vectors has underscored the importance of scalable, high-yield manufacturing methods. Previous work from this group reported the use of recombinant herpes simplex virus type 1 (rHSV) vectors to produce rAAV in adherent HEK293 cells, demonstrating the capacity of this system and quality of the product generated. Here we report production and optimization of rAAV using the rHSV system in suspension HEK293 cells (Expi293F) grown in serum and animal component-free medium. Through adjustment of salt concentration in the medium and optimization of infection conditions, titers greater than $1 \times 10^{14}$ vector genomes per liter (VG/liter) were observed in purified rAAV stocks produced in Expi293F cells. Furthermore, this system allowed for high-titer production of multiple rAAV serotypes $(2,5$, and 9$)$ as well as multiple transgenes (green fluorescent protein and acid $\alpha$-glucosidase). A proportional increase in vector production was observed as this method was scaled, with a final 3-liter shaker flask production yielding an excess of $1 \times 10^{15}$ VG in crude cell harvests and an average of $3.5 \times 10^{14}$ total VG of purified rAAV9 material, resulting in greater than $1 \times 10^{5} \mathrm{VG} /$ cell. These results support the use of this rHSV-based rAAV production method for large-scale preclinical and clinical vector production.

Keywords: AAV vectors production, gene therapy, suspension cell lines, herpes simplex virus

\section{INTRODUCTION}

RECOMBINANT ADENO-ASSOCIATED VIRAL (RAAV) VECTORS have become a powerful research and clinical tool because of their ability to provide in vivo long-term gene expression. ${ }^{1}$ As the uses for rAAV grow, so does the need for large-scale manufacturing methods capable of generating high titers of high-quality vector, not only to meet the ever-expanding needs for preclinical studies and clinical trials, but also the strict quality standards established by the U.S. Food and Drug Administration for a gene therapy drug in compliance with current Good Manufacturing Practice (cGMP; 21 CFR). Yet the widespread full-scale clinical use of rAAV, in view of commercialization, has long been hampered by manufacturing limitations that have appeared as one of the greatest challenges undermining the production of large quantities of rAAV needed for clinical trials. ${ }^{2}$

At present, three main methods are used for rAAV production: transfection, producer cell lines, and viral infection. ${ }^{2}$ Most transfection protocols rely on double- or triple-plasmid transfection of adherent HEK293 cells and are considered not scalable because of the linear increase of flat surface for cell culture. It has now been widely accepted in the field that suspension culture is the only scalable cell culture platform for rAAV manufacturing. Alternatives to HEK293 transfection on flat stocks used suspension-adapted HEK293 cells combined with a transfection process. ${ }^{3-5}$ Using polyethylenimine transfection of suspension-adapted HEK293 cells, Grieger and

${ }^{*}$ Correspondence: Dr. Nathalie Clément, Department of Pediatrics, Powell Gene Therapy Center, 1200 Newell Drive, Academic Research Building, RG-187, University of Florida, Gainesville,FL 32610. E-mail: nclement@peds.ufl.edu

(c) Laura Adamson-Small, et al., 2017; Published by Mary Ann Liebert, Inc. This Open Access article is distributed under the terms of the Creative Commons Attribution Noncommercial License (http://creativecommons.org/licenses/by-nc/4.0/) which permits any noncommercial use, distribution, and reproduction in any medium, provided the original author(s) and the source are credited. 
collaborators reported production of greater than $1 \times 10^{14}$ vector genomes per liter (VG/liter) of cell crude harvest, resulting in approximately $3 \times 10^{13}$ purified rAAV vector. ${ }^{6}$ The method was shown to be adaptable to multiple rAAV serotypes in a fully scalable method. Alternatively, stable cell line production relies on the introduction and selection of cells containing either the AAV rep and cap genes or the rAAV genome of interest. ${ }^{7,8}$ The main advantage of this method is the increased probability that each cell may become an rAAV production center, especially when combined with high transfection or infection efficiency. Detailed publications are somewhat lacking, and therefore the true potential of this method remains to be validated in multiple laboratories and at commercial scale. It is believed that the method is capable of generating high-titer AAV preparations with a possible reduction of the amount of empty capsids. Among some of the potential issues with cell line derivatives is the vector or insert integration loss during prolonged passaging of the cells and the potential for residual adenoviral particles in rAAV products, ${ }^{9,10}$ when adenovirus is used. Last, methods based on viral infection for rAAV production have been developed using either baculovirus or herpes simplex virus type 1 (HSV). The concept of both methods is relatively similar, with rAAV production triggered in the host cells (insect cells or mammalian cells, respectively) on coinfection with two or more recombinant viruses carrying the various AAV regions, such as the AAV genome, AAV rep, and AAV cap. In both systems, the shuttle virus, BEV (baculovirus expression vector) or HSV, provides the helper functions required for rAAV replication and packaging. ${ }^{2,11}$ The main advantage of the infection-based production platforms is the ability to use suspension cultures to streamline the scalability toward hundreds of liters of production harvests.

Previously, we reported an optimized protocol for high-yield production of rAAV9 stocks at high titers, using HEK293 flat stocks. ${ }^{12}$ This protocol generated high-quality material, representing an approximately 5- to 10-fold increase in AAV yield when compared with transfection-made material. Furthermore, the protocol used medium containing 5\% fetal bovine serum (FBS). Production of AAV in suspension format has already been described using baby hamster kidney cells (BHK21), demonstrating the feasibility of this platform for scale-up. However, the published production protocol also reported the use of medium containing $10 \%$ FBS or required a change to serum-free medium $4-6 \mathrm{hr}$ after recombinant HSV type 1 (rHSV) infection, both resulting in overall yields that were less than those reported by our team using the adherent 293 platform. ${ }^{12,13}$ One study reports rAAV production using the rHSV BHK suspension platform in serum-free medium, but vector yields and a detailed description of the manufacturing methods were not described. ${ }^{14,15}$

In this work, we report an optimized scalable production method that uses suspension-adapted HEK293 cells combined with a serum- and animal component-free medium that enables production of high-titer, high-potency, and high-quality rAAV vector preparations in a Good Laboratory Practice (GLP)/GMP-compatible overall process.

\section{MATERIALS AND METHODS \\ Cell lines}

Suspension BHK21C13-2P (BHK) cells were obtained from Sigma-Aldrich (St. Louis, MO) and maintained in Dulbecco's modified Eagle's medium (DMEM; HyClone/GE Healthcare Life Sciences, Logan, UT) supplemented with 5\% FBS (Corning, Corning, NY) and 1\% antibiotic/antimycotic (Gibco/ Thermo Fisher Scientific, Waltham, MA). BHKSFX cells were created by gradual medium exchange into serum-free SFM4Transfx-293 over six passages (HyClone/GE Healthcare Life Sciences). BHK-SFX cells were passaged three times in $100 \%$ serum-free medium before rAAV production. Expi293F cells were purchased from Thermo Fisher Scientific and maintained in Expi293 medium (Thermo Fisher Scientific). V27 cells (a gift from D. Knipe, Harvard, Cambridge, MA) and C12 cells (a gift from P. Johnson, Children's Hospital of Philadelphia, Philadelphia, PA) were maintained in DMEM supplemented with 5\% FBS and Geneticin (50 $\mu \mathrm{g} / \mathrm{ml}$; Sigma-Aldrich).

\section{rHSV construction and production}

Recombinant herpesviruses for rHSV-GFP, rHSVAAV2, and rHSV-AAV9 were generated by homologous recombination as previously reported. ${ }^{12,16,17}$ Recombinant HSV-AAV5 was generated by inserting the AAV2/5 expression cassette obtained from plasmid pXYZ5, using $X b a \mathrm{I}$, into plasmid pHSV106-MCS (previously described ${ }^{12}$ ), linearized

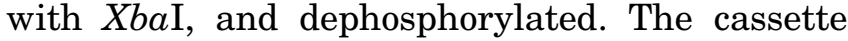
contains the AAV2 p5 promoter sequence, the AAV2 Rep open reading frame (ORF), and AAV5 VP ORF. Ligated DNA was transformed into XL10Gold competent bacteria (Agilent Technologies, Santa Clara, CA), and positive clones were selected on DNA minipreparations. Recombinant HSV-DesGAA was constructed from plasmid pTR-Des-hGAA 
carrying the human acid $\alpha$-glucosidase (GAA) gene under the control of the promoter/enhancer sequence of the human DES (desmin) gene, ${ }^{18}$ flanked by the AAV2 inverted terminal repeats. The entire AAV genome cassette $(A s e \mathrm{I}$ and $P v u \mathrm{I})$ was ligated into the unique SwaI site of plasmid pHSV106MCS and transformed into XL10-Gold competent bacteria, followed by clone screening by DNA minipreparations. Recombinant HSV-AAV5 and HSV-Des-GAA were generated by homologous recombination on transfection into V27 cells as previously described. ${ }^{12}$ rHSV stocks were produced on V27 cells at approximately 80-90\% confluency $(\sim 7-$ $9 \times 10^{8}$ cells per 10 -chamber CellSTACK [CS10; Corning]). rHSV was added to the medium at a multiplicity of infection (MOI) of 0.15 plaqueforming units (PFU)/cell. At 72-96 hr postinfection, cells were lysed in $0.6 \mathrm{M} \mathrm{NaCl}$ (Lonza, Allendale, $\mathrm{NJ}$ ) and harvested, and cell debris was removed by centrifugation. rHSV-containing lysate was concentrated approximately 10 -fold by tangential flow filtration (TFF) (TangenX Sius-300 kDa, Cat. No. XP300L01L; TangenX, Shrewsbury, MA). rHSV stocks were supplemented with glycerol to a $5 \%$ final concentration (Thermo Fisher Scientific), aliquoted, and stored frozen at $-80^{\circ} \mathrm{C}$.

\section{HSV-specific assays}

Plaque assay. rHSV infectious titers were assessed by a traditional plaque assay. V27 cells were seeded at $1.2 \times 10^{5}$ cells per well in 24 -well plates (Corning). Serial dilutions of the rHSV stocks were added to the cells and incubated for $1.5-2 \mathrm{hr}$. The inoculate was removed and DMEM containing $0.8 \%$ agar (low melting temperature [LMT]; Invitrogen/Thermo Fisher Scientific) was poured in each well. Plaques were counted by microscopic examination $72 \mathrm{hr}$ postinfection.

\section{rAAV production and purification}

AAV production. Expi293F or BHK cells were coinfected at the indicated MOI with rHSV stocks containing a gene of interest (encoding green fluorescent protein [GFP] or GAA) and a second rHSV containing AAV Rep2Cap2, Rep2Cap5, or Rep2Cap9. Cells were grown in shaker flasks at $37^{\circ} \mathrm{C}$, $80 \%$ humidity, and 100 and $125 \mathrm{rpm}$ for BHK and Expi293F cells, respectively. Unless noted, cells were infected for $48 \mathrm{hr}$ under conditions similar to those for cell growth and were collected by centrifugation. Infections at volumes greater than 1 liter were grown at speeds reduced to $100 \mathrm{rpm}$. Cell pellets were lysed and analyzed as either crude preparations or purified as described below. For medium supplementation experiments, cells were added to shaker flasks in $80 \%$ of the final medium volume and supplemented with the additional $20 \%$ of medium containing sodium chloride, FBS, or glycerol, or a combination of the three, at the reported concentrations. Adherent HEK293 rAAV production was performed as previously described. ${ }^{12}$

AAV9 purification. For small-scale optimization, cells were pelleted by centrifugation and lysed in a $50 \mathrm{~m} M$ Tris and $150 \mathrm{~m} M$ sodium chloride lysis buffer at $\mathrm{pH}$ 8.5. After three freeze-thaw cycles, lysates were treated with Benzonase for $30 \mathrm{~min}$ at $37^{\circ} \mathrm{C}$ and clarified by centrifugation. For large-scale purification, AAV was purified from cell pellets as previously described. ${ }^{19}$ Briefly, cells were lysed and lysates were digested with Benzonase (EMD Millipore, Billerica, MA) (crude lysate), clarified by protein flocculation and centrifugation, and purified by anion-exchange chromatography using HiPrep SP FF (GE Healthcare Life Sciences, Pittsburgh, PA). Virus was eluted as a discrete peak in a final formulation of $22 \mathrm{~m} M$ sodium citrate, $13.1 \mathrm{~m} M$ citric acid, and $200 \mathrm{~m} M$ sodium chloride, at approximately $\mathrm{pH} 4.8$ (SP peak or purified bulk), and sterile filtered. The virus was further concentrated by tangential flow filtration, using a $100 \mathrm{~K}$ nominal molecular weight cutoff (NMWC) MidGee hollow fiber cartridge (GE Healthcare Life Sciences) and filter sterilized (final stock). Alternatively, purified bulks were concentrated with Apollo 20-ml 150-kDa concentrators (Orbital Biosciences, Topsfield, MA).

\section{rAAV-specific assays}

Vector genome titer. Vector genome titers were obtained by quantitative real-time PCR (Q-PCR) as previously described. ${ }^{12}$ Vector stocks were subjected to RNase-free DNase I digestion $(20 \mathrm{U} / \mathrm{ml}, 30 \mathrm{~min}$, $37^{\circ} \mathrm{C}$ ) (New England BioLabs, Ipswich, MA) and diluted six times in RNase/DNase-free water (Gibco/ Thermo Fisher Scientific), using a 5-fold dilution scheme $\left(5 \times 10^{2}\right.$-fold to $1.56 \times 10^{6}$-fold $)$. PCRs were prepared according to the manufacturer's instructions: $1 \times \mathrm{iQ}$ SYBR green supermix (Bio-Rad, Hercules, CA); $200 \mathrm{n} M$ concentrations of forward and reverse primers; $95^{\circ} \mathrm{C}$ for $10 \mathrm{~min}, 95^{\circ} \mathrm{C}$ for $10 \mathrm{sec}$, $62^{\circ} \mathrm{C}$ for $30 \mathrm{sec}, 40$ cycles (CFX Connect real-time system; Bio-Rad). For rAAV-GFP, primers UF5-3F (5'-CCAGGTCCACTTCGCATATT) and UF5-3R (5'GCGTGCAATCCATCTTGTTC) were used and the standard curve was prepared with our plasmid pTRUF5 (AAV-GFP), as previously described. ${ }^{12}$ For AAV-Des-GAA, desmin promoter-specific primers DES-F (5'-AAGCTTATCAGACCCTTTCTGG) and DES-R (5'-CTCTTTCAGCCCTGGTTATCTC) were 
used in combination with a standard curve prepared with pTR-Des-GAA plasmid. Plasmids were produced at Aldevron (Fargo, ND)

Transduction assay. Adenovirus serotype 5 (Ad5)-infected C12 cells were transduced in 96-well plates with serial dilutions of the rAAV-containing samples. After $42-48 \mathrm{hr}, \mathrm{GFP}$-expressing cells were visually counted by microscopy and titers were calculated.

Identity and purity assay. Purity and capsid amount were assessed by Coomassie staining. Stocks were diluted to $1 \times 10^{13} \mathrm{VG} / \mathrm{ml}$ in rAAV9 excipient (elution buffer) and $2 \times 10^{11} \mathrm{VG}$ was separated on a $10 \%$ sodium dodecyl sulfate (SDS)-polyacrylamide gel (Bio-Rad). Each gel was stained with GelCode Blue (Pierce Biotechnology/Thermo Fisher Scientific, Rockford, IL) and scanned. Purity was assessed by quantification of VP1/2/3 and expressed as a percentage of total protein detected and quantified with the imaging software Quantity One (Bio-Rad).

Western blotting. Purified vector preparations were prepared as for Coomassie staining except that $1 \times 10^{10}$ VG was separated on a $10 \%$ SDS-polyacrylamide gel (Bio-Rad) and transferred to nitrocellulose membrane. AAV capsids were detected with an anti-AAV capsid antibody (diluted 1:2000; American Research Products, Waltham, MA) and a IRDye CW800 goat anti-mouse secondary antibody (Li-Cor) (diluted 1:10,000). Blots were visualized and quantified with an infrared imaging system and accompanying software (Li-Cor, Lincoln, NE).

Flow cytometry. Expi293F cells were infected at an MOI of 2 with rHSV-GFP alone or coinfected with both rHSV-GFP and rHSV-AAV9 at an MOI of 2 and 4, respectively. At $48 \mathrm{hr}$ postinfection, cells were collected by centrifugation, washed in phosphate-buffered saline (PBS), and suspended in $1 \%$ paraformaldehyde in PBS. Cell sorting for GFP expression was performed on a FACSCalibur (BD Biosciences, San Jose, CA) and analyzed with CellQuest version 3.3. Each experiment was run with 10,000 cells, and uninfected Expi293F cells were used as a control for comparison. Total GFPpositive cells and GFP intensity were measured.

\section{Statistical analysis}

GraphPad Prism software (GraphPad, La Jolla, CA) was used to analyze differences between groups, using one-way analysis of variance followed by Dunnett's post-hoc test for group comparison. $p$ values less than 0.05 were considered statistically significant.

\section{RESULTS}

Increased sodium chloride concentration improves rHSV-based rAAV titers in Expi293F suspension cells

This protocol for the production of rAAV9 in suspension was built on the one previously developed for adherent HEK293 cells with the following changes: Expi293F cells were counted and homogenized in fresh medium at a concentration of $1 \times 10^{6}$ cells $/ \mathrm{ml}$ on the day of infection. The inoculum containing the rHSV stocks (rHSV-GFP [UF5], and rHSV-AAV9) in appropriate amounts was prepared in the growth medium and directly added to the cell suspension culture. As for the adherent cell protocol, rAAV production was carried out for $48 \mathrm{hr}$, cells were harvested by centrifugation, and the medium was discarded. Optimization steps were performed at a small scale $(50-\mathrm{ml}$ cell suspension, $5 \times 10^{7}$ cells) by directly analyzing rAAV vector genome and infectious titers from the cell crude lysates to enable quick screening of the various parameters assessed.

During preliminary optimization steps, it was observed that despite maintaining the MOI across the experiments, the yields of rAAV9-GFP vector varied substantially depending on the rHSV stocks used, with rHSV stocks at lower titers often resulting in higher rAAV9 yields. Because this was consistently observed across multiple lots of rHSV, it was ruled out that the relatively high variability of the PFU assay ( $\pm 50 \%$ ) would be the explanation, although it may have contributed. It was then reasoned that because lower rHSV titer resulted in increased volume of each of the rHSV stocks added to the inoculum, a component of the rHSV stock matrix may impact rAAV production. rHSV stock matrix is composed of $1 \times \mathrm{DMEM}$ containing $5 \%$ FBS, 5\% glycerol, and $600 \mathrm{~m} M$ sodium chloride ( $\mathrm{NaCl})$ (see Materials and Methods). To evaluate the direct impact of the rHSV matrix on rAAV production, suspension Expi293F cells were coinfected with constant volumes of the "viral inoculum" containing rHSV-GFP and rHSV-AAV9 at an MOI of 2:4, respectively, but varying volumes of a mock matrix (matrix inoculum) prepared with $1 \times$ DMEM, 5\% FBS, 5\% glycerol, and $600 \mathrm{mM}$ $\mathrm{NaCl}$. The matrix inoculum was supplemented so that the volume of the viral inoculum plus the matrix inoculum would represent $5,10,15,20$, or $25 \%$ of the total growth medium (vol:vol) in the flask. Interestingly, infection in $\mathrm{rHSV}+$ matrix inoculum at a final concentration of $5-15 \%$ of the total volume resulted in a significant increase in rAAV production, with more than a 5 -fold increase in rAAV total infectious units obtained at $10 \%$ final 

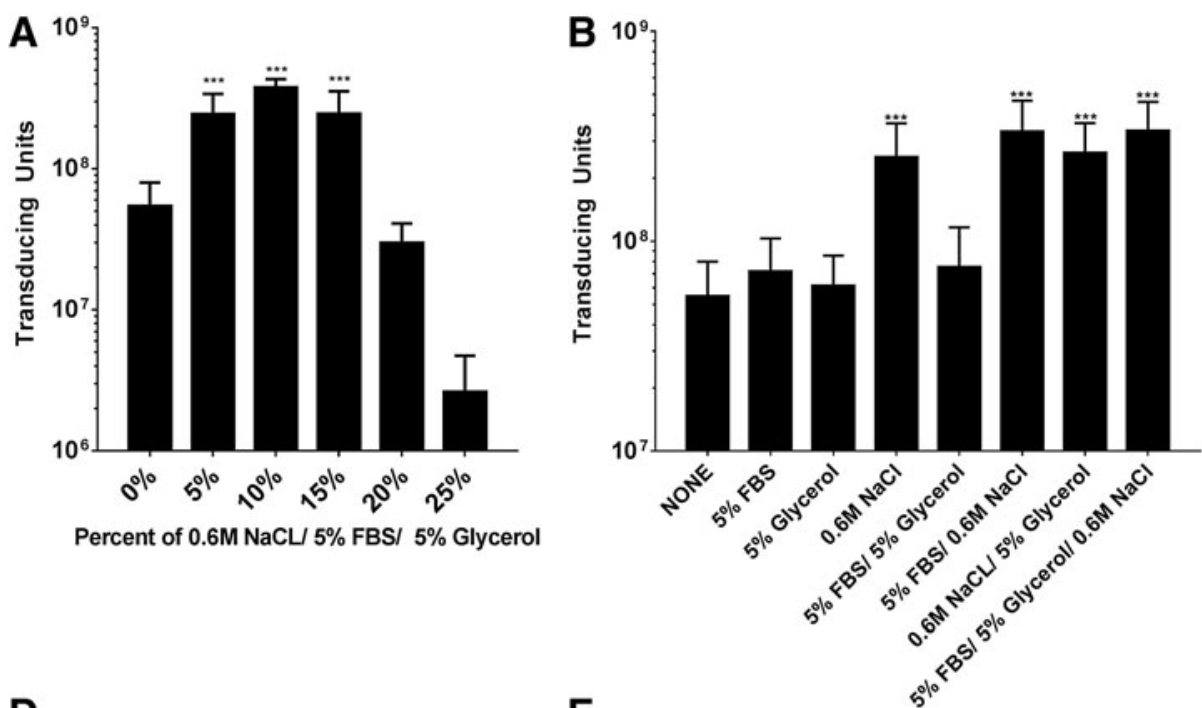

C

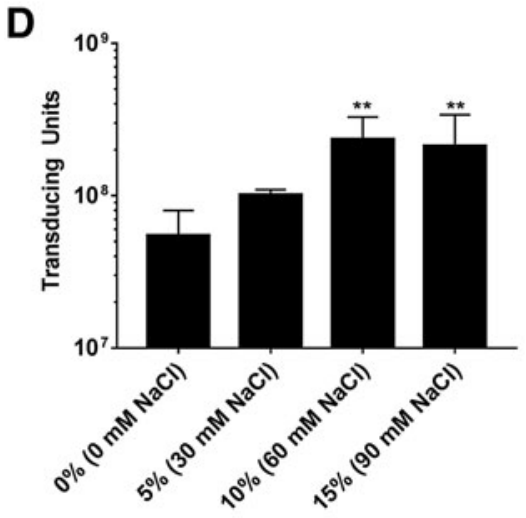

E

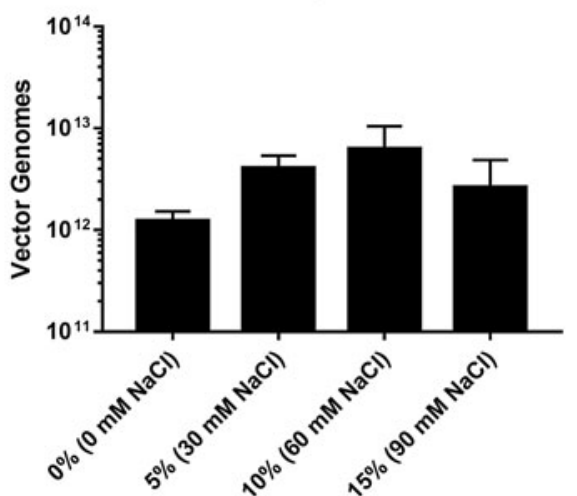

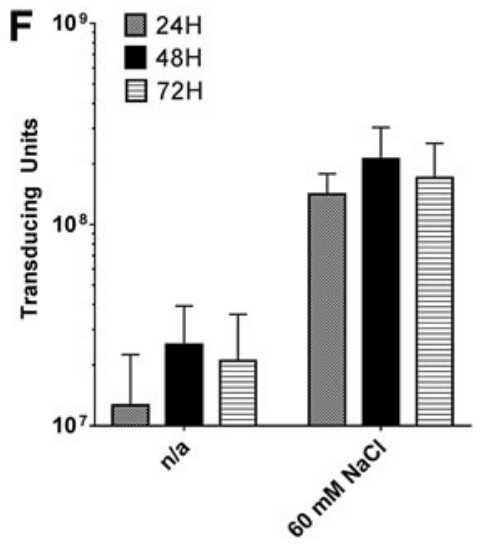

Figure 1. Increased sodium chloride concentrations result in improved rAAV production after rHSV coinfection. (A and B) Transducing units measured in cell lysates $48 \mathrm{hr}$ postinfection in 50-ml working volumes, with the medium supplemented with increasing percentages of the HSV matrix (5\% FBS/5\% glycerol/ $600 \mathrm{mM} \mathrm{NaCl}$ ), or each component individually or in various combinations at a final concentration of $15 \%$ of total medium volume. (C-E) Increased rAAV9 capsid proteins, transducing units, and vector genomes measured in cell lysates $48 \mathrm{hr}$ postinfection, in the presence of $0-90 \mathrm{~m} M$ salt supplementation. (F) rAAV9-GFP production in the absence or presence of salt 24,48 , and $72 \mathrm{hr}$ postinfection. ${ }^{* *} p<0.01,{ }^{* * *} p<0.001$.

rHSV matrix concentration when compared with the same condition not supplemented with matrix inoculum (Fig. 1A). On adjusting the rHSV inoculum with the HSV-free matrix to a final concentration of the total production volume, the "HSV matrix" inoculum added to the cell culture was at $0.5 \%$ FBS, $0.5 \%$ glycerol, and a $60 \mathrm{~m} M$ final concentration of sodium chloride at the time of rHSV infection. Concentration of the rHSV matrix above $20 \%$ of total volume resulted in a net decrease in rAAV production, suggesting a drastic impact on cell metabolism or viability, which was not further characterized.

The next aim was to determine more specifically which of the three main components of the HSV vector matrix (i.e., FBS, glycerol, or salt), or which combination of these components, was responsible for the increase in rAAV production. The same procedure design as described previously was followed, with Expi293F cells infected at an MOI of
2:4 (constant viral inoculum volume) but supplemented with various ratios of matrices made of $1 \times$ Expi293 growth medium supplemented with either 5\% FBS, 5\% glycerol, or $600 \mathrm{~m} M$ sodium chloride alone, or with a combination of each component. Each of the different matrices was added to the production culture to reach a final concentration of $15 \%$ of the final volume. All medium supplementations containing sodium chloride showed an increase in rAAV production that was similar to increases seen when all three components were added, regardless of whether this was prepared in Expi293 medium or DMEM (4.5- to 6.5-fold; Fig. 1B; DMEM results not shown). A similar experiment was performed in which all components were diluted in $1 \times$ DMEM instead of Expi293F medium. The results obtained were comparable to those shown in Fig. 1B, suggesting the components of DMEM had little impact on rAAV production. Together, this demonstrated that $60-90 \mathrm{~m} M$ sodium 
chloride in the production medium resulted in a significant increase in rAAV production when using the rHSV coinfection method in Expi293F cells.

The dose-response curve study was repeated using only sodium chloride: Expi293F cells were coinfected in the presence of increasing amounts of sodium chloride alone, ranging from 30 to $90 \mathrm{mM}$ of supplemented concentrations. A 2- to 4.3-fold increase was seen in total capsid proteins, transducing units, and vector genomes when medium was supplemented at the optimal concentration of $60 \mathrm{~m} M$ sodium chloride (Fig. $1 \mathrm{C}-\mathrm{E}$ ). Total vector genome and transducing unit values are shown in Table 1. This further confirmed that the presence of other additives (FBS, glycerol) was not impacting the effect of sodium chloride on vector production when tested at lower or higher concentrations. To evaluate whether increased salt concentrations damaged cells or resulted in the release of rAAV into the supernatant, virus production in cell pellets and supernatants was evaluated 24,48 , and $72 \mathrm{hr}$ after infection in either medium alone or medium supplemented with $60 \mathrm{~m} M$ sodium chloride. There was no significant difference in total transducing units obtained from lysis of cell pellets at any of the time points (Fig. 1F). Measurement of transducing units in the supernatant demonstrated that less than $2 \%$ of total virus produced was released outside of cells at 24 to $72 \mathrm{hr}$ posttransduction (data not shown).

Various MOIs and ratios between the two rHSVs were tested, keeping in mind that, based on observations described previously, the concentration of sodium chloride may impact the assessment of MOI directly related to the volume of rHSV stocks added to the production medium. Therefore, infection with a combination of MOIs including 1,2 , and 4 for rHSV-GFP and 2, 4, 8, 10, and 12 for rHSV-AAV9, with sodium chloride adjusted to the optimal concentration ( $10 \%$ or $60 \mathrm{mM}$ ), was performed. No major differences were observed with any combination of rHSV coinfection in the MOI range studied (data not shown; $p>0.6$ for all). Results indicated that an MOI of 1-2 for the rHSV-
AAV and an MOI of 4-10 were optimal with regard to rAAV titer. On optimization, all production runs in this study were performed using an MOI of 2:4 for HSV-GFP:HSV-AAV9, respectively. This is also in line with MOIs published for suspensionadapted BHK (sBHK) cells. ${ }^{13}$ This suggests an improvement with increased MOI of virus may be due in part to the increased salt concentration in the medium (obtained from using a higher volume of $\mathrm{rHSV}$ vector), not to the increase in total vector particles.

The next aim was to explore at what point during rHSV infection or the rAAV production cycle was the addition of sodium chloride critical for increased vector production. After infection with rHSV-GFP alone or coinfection with rHSV-GFP and rHSV-AAV9, Expi293F cells were analyzed $48 \mathrm{hr}$ posttransduction by flow cytometry. There was no significant difference in GFP-positive cells, with greater than $95 \%$ of infected cells expressing GFP in both normal and sodium chloridesupplemented cultures (Supplementary Fig. S1A; supplementary data are available online at http:// online.liebertpub.com/hgtb). An approximate 2- to 3 -fold increase in GFP expression was observed in cells infected with rHSV-GFP alone and those infected with both rHSV-GFP and rHSV-AAV9 in the presence of sodium chloride as measured by flow cytometry (Supplementary Fig. S1B). Together, these data strongly suggest that the increase in rAAV titer is not directly correlated to an increase in rHSVmediated cell transduction but instead impacts viral gene expression and/or production of rAAV.

Last, a time course experiment was performed in which salt was added at various time points and/or removed at various time points. Expi293F cells were coinfected with rHSV-AAV9 and rHSV-GFP at an MOI of 2:4, respectively. Cells were infected in normal Expi293 medium or medium supplemented with $60 \mathrm{~m} M$ sodium chloride and either incubated for $48 \mathrm{hr}$ without medium change, or after 4-6 hr, medium was changed to either regular or sodium chloride-containing Expi293 medium. Each combination represents either the presence

Table 1. Impact of sodium chloride on production of multiple rAAV serotypes

\begin{tabular}{|c|c|c|c|c|c|c|}
\hline & \multicolumn{2}{|c|}{ IAAV2-GFP } & \multicolumn{2}{|c|}{ IAAV5-GFP } & \multicolumn{2}{|c|}{ rAAVg-GFP } \\
\hline & Expi293 medium & Medium $+60 \mathrm{mM} \mathrm{NaCl}$ & Expi293 medium & Medium $+60 \mathrm{mM} \mathrm{NaCl}$ & Expi293 medium & Medium $+60 \mathrm{mM} \mathrm{NaCl}$ \\
\hline Total VG & $9.31 \times 10^{11}$ & $2.32 \times 10^{12}$ & $3.14 \times 10^{12}$ & $1.10 \times 10^{13}$ & $1.64 \times 10^{12}$ & $8.92 \times 10^{12}$ \\
\hline VG/cell & $1.86 \times 10^{4}$ & $4.65 \times 10^{4}$ & $6.29 \times 10^{4}$ & $2.20 \times 10^{5}$ & $3.29 \times 10^{4}$ & $1.78 \times 10^{5}$ \\
\hline Total TU & $2.43 \times 10^{10}$ & $1.06 \times 10^{11}$ & $1.88 \times 10^{7}$ & $9.75 \times 10^{7}$ & $4.48 \times 10^{7}$ & $2.20 \times 10^{8}$ \\
\hline TU/cell & 485 & 2110 & 0.38 & 1.95 & 0.90 & 4.40 \\
\hline Ratio VG:TU & 40.26 & 26.74 & $1.76 \times 10^{5}$ & $1.14 \times 10^{5}$ & $4.39 \times 10^{4}$ & $4.05 \times 10^{4}$ \\
\hline
\end{tabular}

GFP, green fluorescent protein; rAAV2, recombinant adeno-associated virus type 2; VG, vector genomes; TU, transducing units. 
or absence of salt at the time of infection only, during viral production only, or during the entire infection and production process. Increases in rAAV9 transducing units and capsid proteins were observed under infection conditions in which increased sodium chloride was present $4-6 \mathrm{hr}$ posttransduction, regardless of whether the medium was changed or the rHSV was left throughout the infection process (Supplementary Fig. S1C). No increase was seen when medium was changed 4$6 \mathrm{hr}$ posttransduction to Expi293 medium without sodium chloride. The need for sodium chloride during viral production, but not entry, was confirmed in 3-liter cultures. Sodium chloride was present either at rHSV infection, added $6 \mathrm{hr}$ postinfection, or added when medium was changed at $6 \mathrm{hr}$ to medium supplemented with $60 \mathrm{~m} M$ sodium chloride. A significant decrease was seen in vector genomes in the cultures where medium changes did occur, but no significant change in vector genomes or transducing units was observed when sodium chloride was added $6 \mathrm{hr}$ after rHSV infection (Supplementary Fig. S1D and E). These data demonstrate that increasing salt concentrations at the time of rAAV production significantly improves viral titers in Expi293F cells and suggest that the salt does not improve HSV entry itself.

Altogether, these data convincingly show that increasing sodium chloride concentration in the medium at the time of rAAV production increases rAAV titers when using the rHSV coinfection method in Expi293. This demonstrates that optimal vector production can be achieved by $48 \mathrm{hr}$ postinfection in cell harvests with minimal release of vector into supernatant.

\section{Sodium chloride-mediated increase in rAAV production occurs in other rAAV serotypes and transgenes and for other cell lines}

Impact of sodium chloride on rAAV production using other rAAV serotypes, other genes of interest, and other cell lines were investigated next. Production of rAAV2-GFP, AAV5-GFP, and rAAV9-GAA was tested in Expi293F cells in medium supplemented with $0-90 \mathrm{mM}$ sodium chloride. For rAAV2-GFP and rAAV5-GFP, there was a 4.3- to 5.2-fold increase in transducing units and a 2.3- to 2.5-fold increase in vector genomes, respectively, when medium was supplemented with $60 \mathrm{~m} M$ sodium chloride (Fig. $2 \mathrm{~A}$ and B). A 3 -fold increase in rAAV9-GAA vector genomes was observed with medium supplemented to $60 \mathrm{~m} M$ with sodium chloride (Fig. 2B). Total vector genome and transducing unit values are shown in Table 1. These data demonstrate that sodium chloride me- dium adjustment improves rAAV production in other serotypes and genes of interest.

Previous work characterizing rHSV coinfection in suspension was performed on BHK cells in DMEM supplemented with FBS. $^{13}$ To further compare viral production and sodium chloride medium supplementation, BHK cells grown in DMEM containing 5\% FBS and BHK cells adapted to growth in SFM4Transfx serum-free medium (BHK-SFX) were infected with rHSV-AAV9 and rHSV-GFP in the presence of increasing amounts of sodium chloride. Adaptation of BHK cells to serum-free medium showed similar replication and growth characteristics as BHK cells grown in DMEM-FBS, and the adaption method is described in Materials and Methods. Similar to Expi293F cells, BHK cells grown in DMEM with 5\% FBS showed a significant, 2.2-fold increase in transducing units when medium was supplemented with $60 \mathrm{~m} M$ sodium chloride $(p<0.001$; Fig. $2 \mathrm{C})$. Interestingly, BHK cells adapted to growth in serumfree medium showed a 5.2-fold increase in rAAV9 transducing units with only $30 \mathrm{mM}$ sodium chloride (Fig. 2C). Total vector genomes were increased 2- to 3.5-fold after supplementation with optimal concentrations of salt in BHK cells grown in DMEM with 5\% FBS and serum-free medium, respectively (Fig. 2D). Both Expi293F and BHK cells grown in serum-free medium without additional sodium chloride produced 1.6- and 5-fold less virus than BHK cells grown in DMEM with 5\% FBS, suggesting lower yields by rHSV in serum-free platforms. Supplementing these serum-free media with salt improved viral production to levels approximately equivalent (BHK-SFX cells) or 2.8-fold greater (Expi293F cells) compared with BHK cells in DMEM-FBS, suggesting addition of sodium chloride in the rHSV production process can improve the lower rAAV yields that can be observed during rAAV production in serum-free medium. ${ }^{3}$ Overall, these data show that increasing sodium chloride concentrations in the rHSV platform improves rAAV vector production across multiple serotypes, transgenes, cell lines, and media.

\section{The production system using Expi293 is highly scalable}

The next aim was to evaluate the scalability of this novel suspension production procedure by evaluating total viral production in crude lysates. Analyzing Benzonase-treated crude lysates is critical to assess total production capabilities independently of the purification processes, which may vary across laboratories and even across the various AAV serotypes. Figure 3 shows the total 

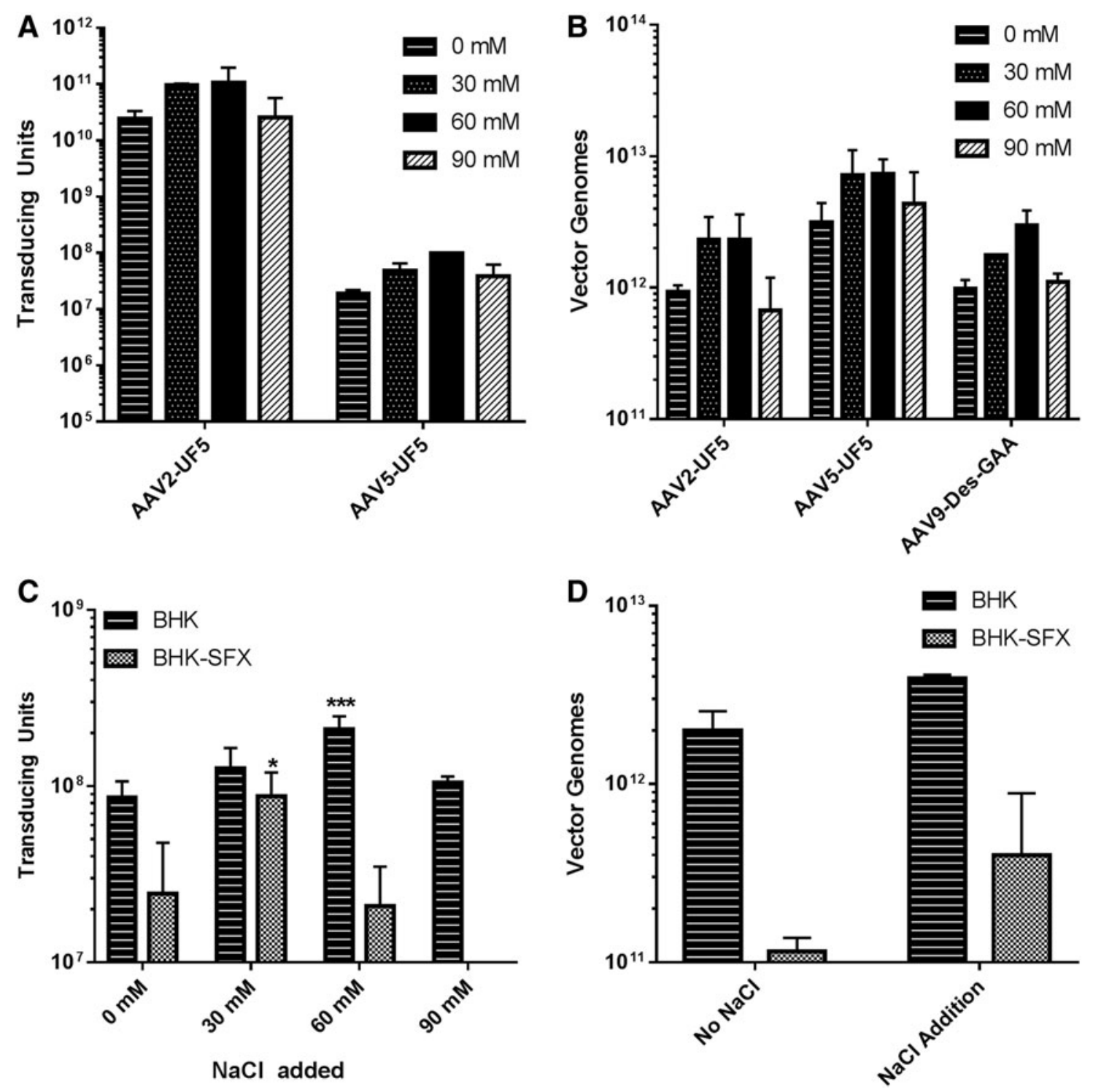

Figure 2. Increased rAAV9 production after sodium chloride supplementation for other rAAV serotypes, genes of interest, and/or cell types. Total transducing units (A) and total vector genomes (B) were evaluated for rAAV2-GFP, rAAV5-GFP, and rAAV9-Des-GAA produced by rHSV coinfection in shaker flasks (50-ml working volume) in the presence of increased sodium chloride concentration ( $n=2$ for all). (C) Impact of increased sodium chloride on rAAV9-GFP production in BHK cells grown in DMEM with 5\% FBS (BHK) or adapted to serum-free medium (BHK-SFX). (D) Total rAAV9-GFP vector genomes produced in BHK and BHK-SFX cells by rHSV coinfection in the absence or presence of optimal salt supplementation (30 and $60 \mathrm{~m} M$, respectively). ${ }^{*} p<0.05$, ${ }^{* * *} p<0.001$.

transducing unit and vector genome yields as a function of working production volumes ranging between $50 \mathrm{ml}$ and 3 liters, or a 60 -fold scaleup. The increase in yield is relatively linear to the volume increase for scales up to 0.5 liter, with an average, approximately 7-fold increase in transducing units when compared with $50 \mathrm{ml}$ (Fig. 3A). However, the yield increase is about twice as much as the increase in volume from $50 \mathrm{ml}$ to 1 liter ( $\sim 40$-fold) and to 3 liters ( $\sim 155$-fold). The highest yields were obtained from 3-liter working volumes for rAAV-GFP, with an average of $1.24 \times 10^{15}$ $\left( \pm 4.66 \times 10^{14}\right)$ VG $(n=4)$ or $4.12 \times 10^{14}\left( \pm 1.55 \times 10^{14}\right)$ VG/liter in crude lysates, an 8 -fold increase as compared with 1 liter, and a 27-fold increase when compared with 0.5 liter (Fig. 3B). This resulted in an average of $4.12 \times 10^{5}\left( \pm 1.55 \times 10^{5}\right)$ VG/cell (Fig. 3C). In contrast, production in a 3-liter culture without the addition of sodium chloride resulted in $1.40 \times 10^{14} \mathrm{VG}$ total production in crude lysates, or $4.63 \times 10^{13}$ VG/liter, a 9.1-fold reduction compared with sodium chloride-supplemented flasks infected in parallel (Supplementary Fig. $\mathrm{S} 1 \mathrm{~F})$. The increase in infectious units paralleled the particle titers, with an average of $3.16 \times 10^{10}$ $\left( \pm 8.90 \times 10^{9}\right)$ TU for the 3-liter crudes as compared with $1.05 \times 10^{9}\left( \pm 5.74 \times 10^{8}\right) \mathrm{TU}(n=4)$ for 0.5 liter, an approximately 30 -fold increase. Yields for 

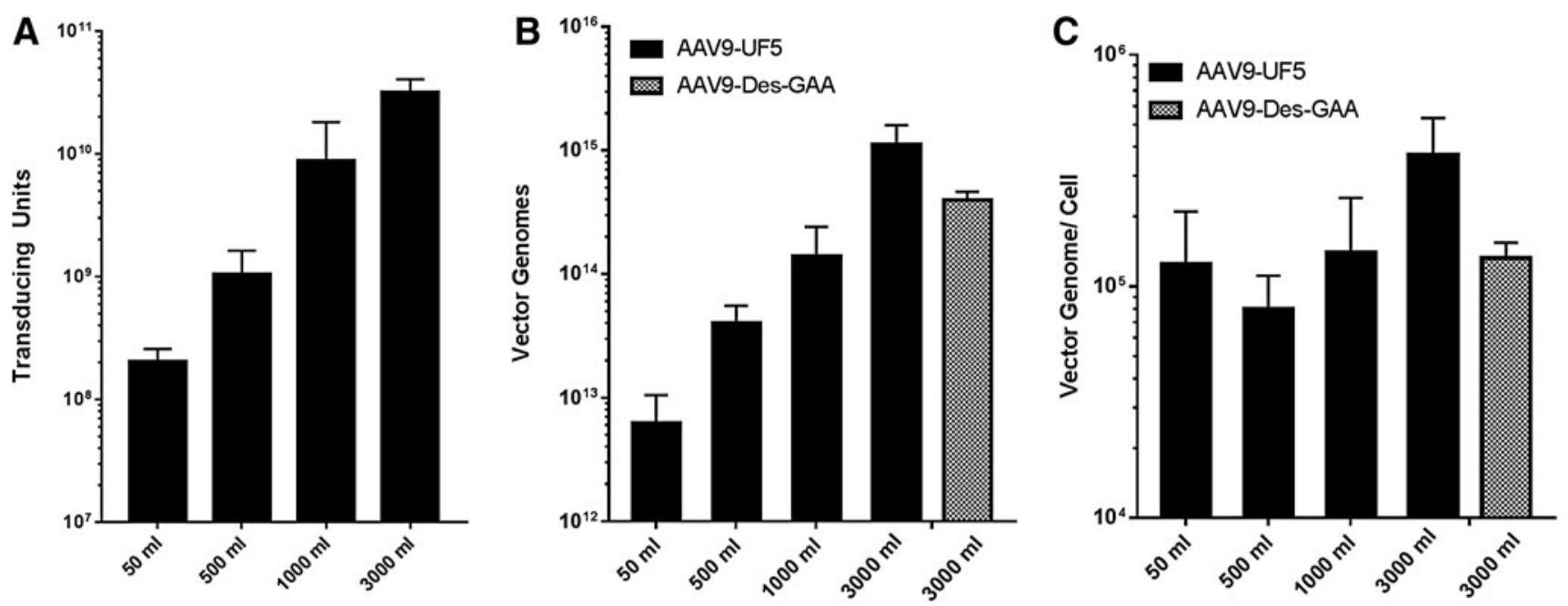

Figure 3. Scalability of rAAV production in Expi293F cells. Transducing units for rAAV9-GFP (A) and total vector genomes for rAAV9-GFP and rAAV9-Des-GAA (B) were assessed in crude lysates from Expi293F cultures ranging from 50 to $3000 \mathrm{ml}$. (C) Total vector genome values are presented as vector genomes per cell at each scale.

rAAV9-GAA were more consistent with an expected scaled-up increase, with an average of $3.97 \times 10^{14}$ total VG/3 liters or $1.2 \times 10^{14}$ VG/liter of culture ( $n=2$; Fig. 3B). A better growth environment in the 5-liter flask, used to produce the 3-liter working production batches, may explain this more favorable scale. The ratio of medium to the flask volume, the shaking speed, and/or the flask shape may promote oxygenation, reduce cell shear, or improve virus-cell contact. As observed at smaller scales, the infectivity of the rAAV9 batches was maintained throughout the scaling process, with a VG:TU ratio of $3.88 \times 10^{4}\left( \pm 1.88 \times 10^{4}\right)$ (11 independent runs at $0.5,1$, and 3 liters).

\section{The biological quality of the rAAV9 vectors produced by the salt-optimized suspension system is maintained}

Although manufacturing yields are often provided from crude harvests to enable side-by-side comparison across different production methods, it is essential to carefully characterize the final product not only to support the results obtained in crude samples, but more importantly to rule out any potential bias from the presence of cellular and viral bioproducts (e.g., HEK293 or HSV proteins). For this purpose, five independent and fully purified vector batches prepared from Expi293F cells were fully characterized with respect to physical and biological parameters. Stocks prepared in the presence or absence of salt supplementation were assessed side-by-side, and compared with preparations that were produced on adherent HEK293 cells by HSV coinfection, as previously described. ${ }^{12}$ Highly concentrated stocks were generated using our GMP-like purification method. ${ }^{19}$
Sodium chloride medium supplementation was also tested in the previously published adherent HEK293 cell, rHSV-based, optimized rAAV9 production method. ${ }^{12}$ When DMEM was supplemented with $60 \mathrm{~m} M$ sodium chloride, there was a significant, 1.6- and 2.2-fold increase in rAAV vector genomes and transducing units, respectively (Fig. 4A and B, solid columns; $p<0.05$ ). Although this confirms that sodium chloride also increases vector production in an adherent HEK293 cell platform, this suggests that the sodium chloride-mediated increase in vector production is more robust in the suspension format. The impact of sodium chloride supplementation was next tested in the traditional transfectionmediated rAAV9 production platform. HEK293 cells were cotransfected with pTR-UF5 (GFP) and pDG-UF9-KanR plasmids and $60 \mathrm{~m} M$ salt was supplemented at the time of transfection or $6 \mathrm{hr}$ posttransfection. ${ }^{12}$ Salt supplementation resulted in a dramatic decrease in rAAV yields (data not shown). This suggested that the impact of sodium chloride may be specific to the rHSV production method, or that further experimental optimization is required to adapt it to the adherent cell format or to other AAV production methods.

Total vector genome yields per production unit for adherent HEK293 and suspension Expi293F cells are reported in Fig. 4. The total yield from five independent salt-optimized suspension runs of rAAV9-GFP in Expi293F cells averaged at $3.5 \times 10^{14}\left( \pm 1.7 \times 10^{14}\right) \mathrm{VG}$ (Fig. $4 \mathrm{~A}$, shaded column) or $1.17 \times 10^{14}\left( \pm 5.66 \times 10^{13}\right)$ VG/liter (Fig. $4 \mathrm{D}$, shaded column) of suspension culture. Overall, the yields in Expi293F cells appeared approximately 3 -fold superior to yields obtained in adherent 

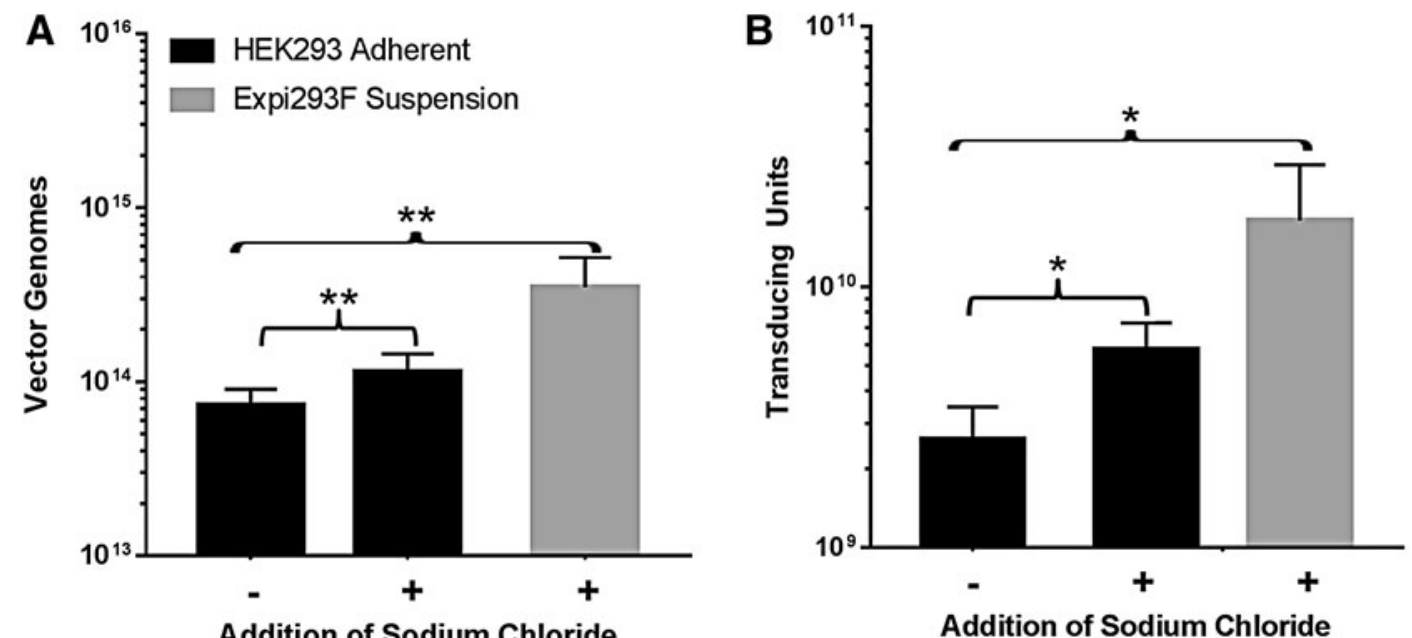

Addition of Sodium Chloride

Addition of Sodium Chloride

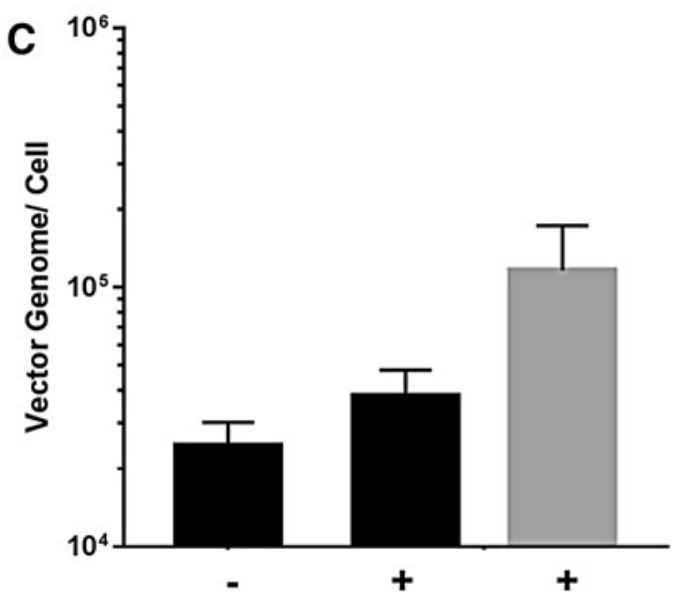

Addition of Sodium Chloride

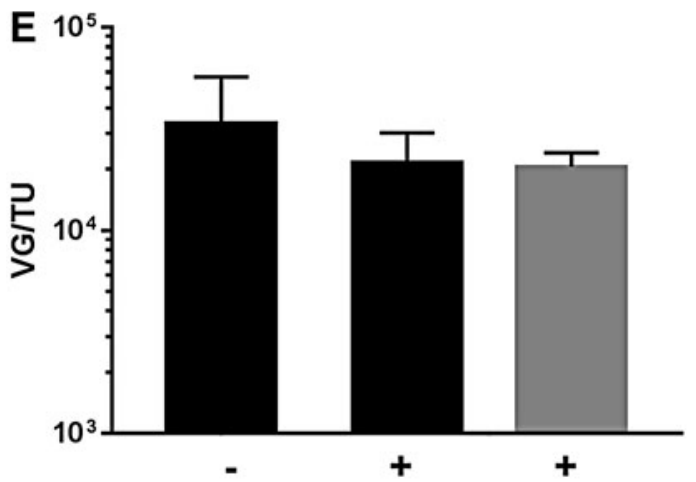

Addition of Sodium Chloride

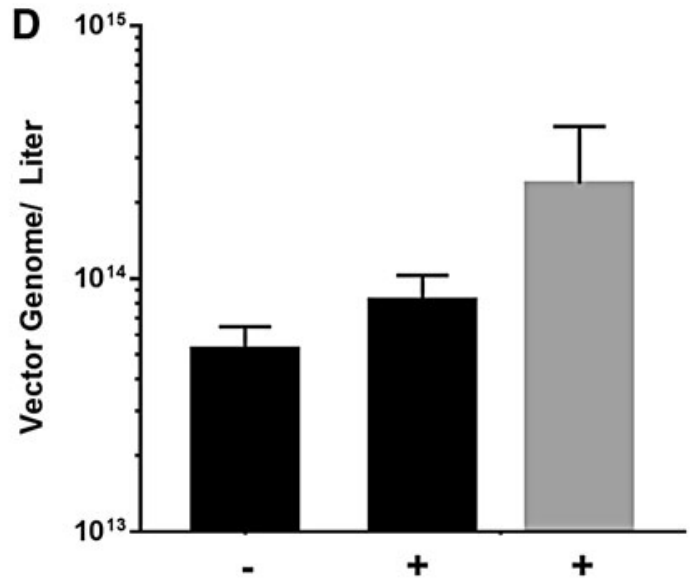

Addition of Sodium Chloride

F

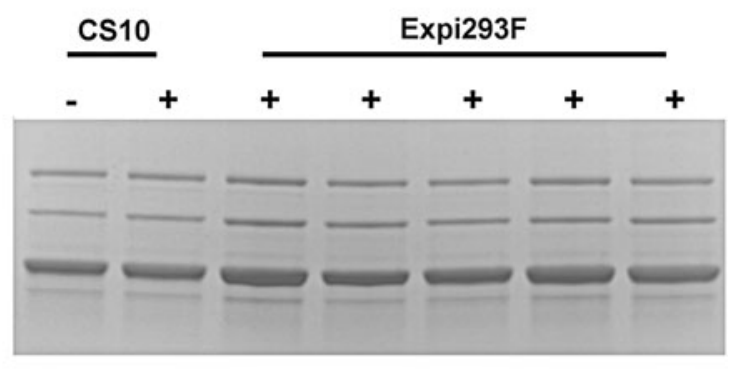

Figure 4. Characterization of rAAV vector made in Expi293F suspension system. (A and B) rAAV9-GFP was produced by rHSV infection in adherent HEK293 cells grown in 10-chamber CellSTACKs (CS10) under optimal conditions (approximately $3 \times 10^{9}$ cells) either in DMEM containing $5 \%$ FBS alone ( $\left.n=4\right)$ or supplemented with $60 \mathrm{~m} M$ sodium chloride $(n=6)$, or in a 3 -liter suspension culture containing $3 \times 10^{9}$ Expi293F cells $(n=5)$. Total vector genome (A) and transducing unit (B) yields are shown. Total purified vector genomes are represented relative to units produced per cell (C) and per liter of medium (D). (E) The vector genome-to-transducing unit (VG/TU) ratio was calculated for each sample, and the averaged values for each group are shown. (F) Coomassie staining of rAAV9-GFP final stocks produced in HEK293 CS10 in the absence of salt supplementation (lane 1), or supplemented with sodium chloride (lane 2), or produced in Expi293F 3-liter cultures supplemented with sodium chloride (lanes 3-7). A total of $10^{11}$ VG was loaded per well. ${ }^{*} p<0.05,{ }^{* *} p<0.01$. 
HEK293 cells infected in the presence of increased sodium chloride concentrations $\left(1.15 \times 10^{14}\right.$ $\left[ \pm 2.97 \times 10^{13}\right]$ VG/CS10; $n=6$; Fig. $4 \mathrm{~A}$ ). This resulted in an average of $1.17 \times 10^{5} \mathrm{VG} /$ cell in Expi293F cells compared with $3.82 \times 10^{4}$ and $2.45 \times 10^{4} \mathrm{VG} /$ cell for the same rAAV9-GFP construct in adherent HEK293 cells with and without sodium chloride supplementation, respectively (Fig. 4C). The final titers for rAAV9-GFP produced in Expi293F cells ranged between $2.6 \times 10^{13}$ and $6.1 \times 10^{13} \mathrm{VG} / \mathrm{ml}$. It is important to note that some of the final stocks produced on HEK293 cells were concentrated with a spin device in lieu of a hollow fiber cartridge (tangential flow filtration). Spin concentration resulted in about $20 \%$ loss of the vector whereas the TFF method supported $100 \%$ recovery of the biological material. This may account for slightly underestimated yields for the HEK293 cells in this study. More importantly, the infectivity of the vector preparations produced with the suspension platform and those produced in increased sodium chloride medium was highly comparable, if not improved, to those of vectors produced using the adherent platform, with an average VG:TU ratio of $2.07 \times 10^{4}$ in Expi293F cells versus $2.18 \times 10^{4}$ and $3.39 \times 10^{4}$ in adherent HEK293 cells with and without sodium chloride supplementation, respectively (Fig. 4E). Therefore, salt supplementation did not affect the infectivity of the rAAV9 particles independently of the production format.

Finally, overall purity and capsid ratios in the fully purified vector preparations were assessed. Identical amounts of vector genomes were analyzed by Coomassie staining and Western blotting. Figure $4 \mathrm{~F}$ shows that the overall capsid load is similar whether the rAAV9 stocks were prepared in Expi293F or HEK293 cells, or in the presence or absence of salt supplementation. This observation was confirmed by Western blot analysis (data not shown). Therefore, the ratio of full-versus-empty capsids is not impacted by the suspension production platform or by the salt supplementation. The purity was greater than $90 \%$ in all cases, with little detection of non-AAV9 byproduct at the amounts tested. A more thorough assessment of the overall stock purity in view of clinical manufacturing is underway.

\section{DISCUSSION}

The increased interest in rAAV as a therapeutic vector and commercial product has highlighted the need for a large-scale manufacturing method for vector production. Whereas rAAV vectors are relatively easy to make for research use, large-scale manufacturing methods that can provide high-titer and high-purity vector continue to lag behind the immense clinical demand. It is not surprising that both industry and academic laboratories, often in collaborative efforts, are poised for an unprecedented investment of resources toward this common goal. This study details an rHSV-based production method that produces high-titer rAAV vectors in a serum- and animal component-free suspension Expi293F cell platform. To our knowledge, it is the first time that a simple component such as sodium chloride has been shown to have a dramatic effect on rAAV production in a suspension platform. With optimal sodium chloride concentrations in the production media, increased vector titers were achieved for multiple rAAV serotypes and transgenes.

The exact mechanism by which sodium chloride increases rAAV production was not fully investigated as part of this current work because the primary goal was to establish an efficient, robust, and attractive method to produce rAAV. Further, little is known or documented in the literature about the specific impact of sodium chloride on rHSV or rAAV viral cycles. Although preliminary, these data shed some light on possible biological mechanisms affected by sodium chloride. Concentrations up to $0.3 \mathrm{M}$ sodium chloride were reported to increase specific binding of the HSV1 KOS strain to GMK-AH1 cells. ${ }^{20}$ However, these data suggest that HSV binding and/or entry may not be significantly impacted. First, a flow cytometric analysis showed that the number of rHSV-GFP-infected Expi293F cells was similar independent of the sodium chloride concentration. Second, it was shown that sodium chloride could be added up to $6 \mathrm{hr}$ after inoculation of the production medium with rHSV, with minimal impact on rAAV production. Interestingly, the flow cytometric analysis did reveal an increase in GFP expression in cells infected in the presence of optimal amounts of sodium chloride. This observation suggests that salt may directly impact either HSV or AAV gene expression, or both. A study with poliovirus showed that increased potassium chloride concentrations at $6-9 \mathrm{hr}$ postinfection resulted in an increase in viral translation. ${ }^{21}$ However, the increase in GFP expression could also be linked to an increase in AAV genome copies on enhanced replication levels, which could result from either increased helper HSV gene expression or AAV rep and cap gene expression in the infected cells. The latter is supported by Western blot analysis showing significantly increased AAV9 capsid expression under salt-optimized conditions. Whether an optimized salt concentration alters the cellular environment and/or improves rAAV production through mechanisms dependent on or 
independent of HSV infection remains to be further studied. Furthermore, the effect of other salts, alone or in combination, is being investigated in our laboratory.

This work also suggests that there may not be one universal optimal sodium chloride concentration to improve AAV production. In fact, it is recommended that each construct, serotype, cell line, and/or growth medium be optimized with regard to salt supplementation. In many cases, the exact final concentration of sodium chloride may not be determined. Hence the sodium chloride concentration of most of the media evaluated in this study could not be obtained from the commercial vendors because the formulations were proprietary, with the exception of DMEM. The reported sodium chloride concentration in DMEM is $109.5 \mathrm{~m} M$. For both BHK suspension and 293 adherent cells grown in DMEM, the highest increase in rAAV vector production was observed when $60 \mathrm{mM}$ sodium chloride was added. This corresponds to a final concentration of $169.5 \mathrm{~m} M$. The concentration of sodium chloride observed in normal saline is $154 \mathrm{~m} M$, suggesting salt supplementation may be ideal when a final concentration is close to normal or physiological levels. Although the sodium chloride molarity in the other media tested here is unknown, this suggests the optimal range for increased rAAV production would fall in the range observed in physiological milieus. Because the natural host environment for both HSV and AAV is the human body, it would be sensible to think that optimal conditions would be those most resembling the natural environment of the viral life cycle.

One of the most attractive data sets from this study is the overall yields obtained when using the HSV system both in adherent and suspension platforms. Here we report yields in crude Benzonase-treated harvests exceeding $3 \times 10^{14}$ VG/liter of production suspension culture, resulting in about $3.5 \times 10^{5} \mathrm{VG} /$ cell. These yields match and even surpass the highest AAV amount currently demonstrated for other suspension methods, which includes the baculovirus expression system (BEV) system, the transfection of suspension-adapted HEK293 cells, and HeLa-derived stable cell lines, as previously discussed. ${ }^{12}$ The BEV system is able to generate approximately $2.4 \times 10^{14}$ VG/liter in crude $S f 9$ cell harvests ${ }^{22}$ - however, because the cell concentration used is 3- to 4-fold higher than the concentration used in our protocol, the amount per cell is significantly reduced to $9 \times 10^{4}$. The HSV infection method using suspension-adapted BHK cells reported about $1-2 \times 10^{14}$ VG/liter in harvests. ${ }^{13}$ Transfection in the suspension system, also using HEK293 cells, reported yields ranging from about $1 \times 10^{14}$ VG/liter or $1 \times 10^{5} \mathrm{VG} /$ cell, also in crude material. ${ }^{6}$ It is unofficially reported that yields may be higher when using stable cell lines. Unfortunately, data are still scarce in the literature and conclusive comparison with our system could not be formally done at this time. ${ }^{9,23}$

When combined with downstream processes, which vary widely depending on the rAAV serotype produced, overall yields of purified material range from $1 \times 10^{13}$ VG/liter for the transfection in suspension HEK293 cells to a maximum of $4-5 \times 10^{13}$ VG/liter for the BEV system. ${ }^{6,22,24,25}$ The first clinical AAV production using the HSV system in sBHK cells was performed by Applied Genetic Technologies Corporation (AGTC, Alachua, FL) with the final yield approaching $2 \times 10^{13} \mathrm{VG} /$ liter or $1 \times 10^{4} \mathrm{VG} / \mathrm{cell}^{26}{ }^{26}$ Yields from the serum-free sBHK protocol have not been published. Preclinical and clinical needs to support ongoing trials appear negligible (less than $5 \times 10^{13}$ total VG for the entire toxicology study or clinical trial) and do not reflect the method capability. ${ }^{14,15}$

Our system is the first to describe a serum-free Expi293 platform for the production of rAAV, using rHSV. Because the rHSV stocks are currently produced in a serum-containing medium, it is possible that residual traces of FBS may still be detected in the final product. However, the high concentration of HSV stocks combined with the low MOIs used in this system do result in an overall input of less than $2 \%$ ( $\mathrm{vol} / \mathrm{vol})$, resulting in a serum concentration of $0.1 \%$ or below. Further, rAAV is purified from PBS-rinsed cells, which should remove the majority of FBSderived impurities. As a matter of fact, the sBHK system also relies on FBS-containing HSV stocks and final test article stocks were shown to contain detectable amounts of bovine serum albumin. ${ }^{14,15}$

Taken together, for a phase 1 clinical study, we calculated the theoretical production scale to produce $1 \times 10^{17}$ VG in crude material, which would equate to approximately $2 \times 10^{16} \mathrm{VG}$ of Investigational New Drug (IND)-enabling purified material (assuming $\sim 20 \%$ recovery from most downstream processes). As much as 1000 liters of cell culture would be required if using the transfection in the suspension HEK293 platform or the HSV/sBHK platform, 400-500 liters if using the BEV system, or 175-250 liters with our Expi293F system. To provide the reader with another perspective related to theoretical scales of AAV manufacturing to support market approval, we extrapolated the theoretical yields of rAAV9 starting from the minimal amount of raw material generated from seven CS10-worth viral seed stock (VSS) (also summarized in Fig. 5). HSV working viral banks ( 2120 


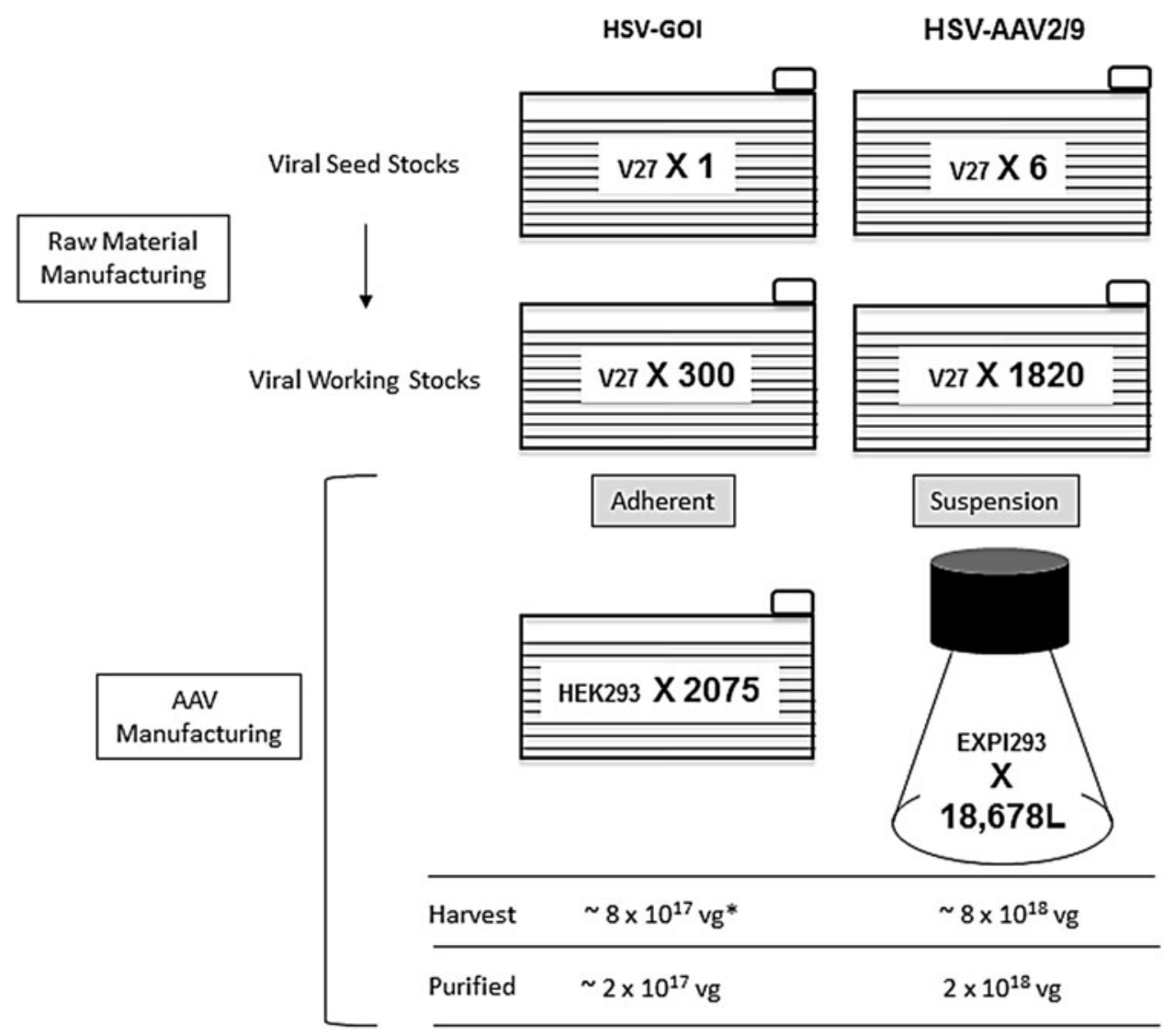

Figure 5. Theoretical HSV system scalability. Extrapolation is based on current average rHSV yields for the constructs discussed in this work. HSV stocks are produced from adherent V27 cells grown in 10-layer flasks (CS10s). Number of production units (CS10s for adherent; liters for suspension), as well as cell type (V27, HEK293, or Expi293F), are indicated for each step and are rounded for clarity. HSV-GOI (gene of interest) and HSV-Rep2Cap9 seed stocks are produced from one and six CS10s, respectively, to support multiplicities of infection (MOls) used in the adherent cell platform (2:12, respectively).

CS10s) would provide raw material in sufficient quantity to infect approximately $2075 \mathrm{CS} 10$ s and generate approximately $8 \times 10^{17} \mathrm{VG}$ in unprocessed production harvests. The same amounts would suffice to infect approximately 18,678 liters of Expi293 and generate about $8 \times 10^{18} \mathrm{VG}$. Although theoretical, and by no means representative of scales currently performed or needed, this exercise emphasizes the future need to implement scalable production methods for the raw materials, in this case rHSVs, for amounts of rAAV above approximately $1 \times 10^{18}$ VG in bulk.

In conclusion, our solid collection of data offers a 3 -fold impact toward enabling manufacturing of large-scale high-titer and high-quality rAAV vectors: (1) a suspension-adapted and highly scalable production platform, (2) serum-free production cultures, and (3) a unique biological quality of the resulting product. Although yields will vary from construct to construct, these data provide a strong framework toward achievable high yields to support preclinical and clinical studies, with an unparalleled flow path toward commercialization- enabling scales. Altogether, this method is poised to facilitate IND-enabling preclinical and clinical studies for AAV-driven gene therapy applications.

\section{ACKNOWLEDGMENTS}

This work was partly supported by the U.S. Department of Defense (W81XWH-13-1-0283 to B.J.B.). The authors thank Dr. Bloom (University of Florida) for constructive discussion related to HSV, Dr. Brian Cleaver for GMP translational process development, the staff of the Powell Gene Therapy Center Vector Core for technical assistance, and Solid GT staff for their generous contribution to laboratory equipment that partly enabled this work.

\section{AUTHOR DISCLOSURE}

M.P. declares no conflict of interest. L.A.-S., B.J.B., and N.C. have a pending patent application related to this research. B.J.B. is an inventor of intellectual property owned by the Johns Hopkins University and the University of Florida related to this research and has AAV patents licensed to various biopharmaceutical companies. 


\section{REFERENCES}

1. Samulski RJ, Muzyczka N. AAV-mediated gene therapy for research and therapeutic purposes. Annu Rev Virol 2014;1:427-451.

2. Clément N, Grieger JC. Manufacturing of recombinant adeno-associated viral vectors for clinical trials. Mol Ther Methods Clin Dev 2016; 3:16002.

3. Hildinger M, Baldi L, Stettler M, et al. High-titer, serum-free production of adeno-associated virus vectors by polyethyleneimine-mediated plasmid transfection in mammalian suspension cells. Biotechnol Lett 2007;29:1713-1721.

4. Chahal PS, Schulze E, Tran R, et al. Production of adeno-associated virus (AAV) serotypes by transient transfection of HEK293 cell suspension cultures for gene delivery. J Virol Methods 2014; 196:163-173.

5. Durocher Y, Pham PL, St-Laurent G, et al Scalable serum-free production of recombinant adeno-associated virus type 2 by transfection of 293 suspension cells. J Virol Methods 2007;144: $32-40$.

6. Grieger JC, Soltys SM, Samulski RJ. Production of recombinant adeno-associated virus vectors using suspension HEK293 cells and continuous harvest of vector from the culture media for GMP FIX and FLT1 clinical vector. Mol Ther 2016;24:287-297.

7. Gao GP, Qu G, Faust LZ, et al. High-titer adenoassociated viral vectors from a Rep/Cap cell line and hybrid shuttle virus. Hum Gene Ther 1998; 9:2353-2362.

8. Liu X, Voulgaropoulou F, Chen R, et al. Selective Rep-Cap gene amplification as a mechanism for high-titer recombinant AAV production from stable cell lines. Mol Ther 2000;2:394-403.

9. Martin J, Frederick A, Luo Y, et al. Generation and characterization of adeno-associated virus producer cell lines for research and preclinical vector production. Hum Gene Ther Methods 2013;24: 253-269.
10. Martin JN, Wolken N, Brown T, et al. Lethal toxicity caused by expression of shRNA in the mouse striatum: implications for therapeutic design. Gene Ther 2011;18:666-673.

11. Bakker A. AAV vector production: baculovirus vs transient transfection. Hum Gene Ther 2008;19: 1067-1068

12. Adamson-Small L, Potter M, Falk DJ, et al. A scalable method for the production of high-titer and high-quality adeno-associated type 9 vectors using the HSV platform. Mol Ther Methods Clin Dev 2016:3:16031.

13. Thomas DL, Wang L, Niamke J, et al. Scalable recombinant adeno-associated virus production using recombinant herpes simplex virus type 1 coinfection of suspension-adapted mammalian cells. Hum Gene Ther 2009;20:861-870.

14. Ye GJ, Budzynski E, Sonnentag P, et al. Safety and biodistribution evaluation in cynomolgus macaques of rAAV2tYF-PR1.7-hCNGB3, a recombinant AAV vector for treatment of achromatopsia. Hum Gene Ther Clin Dev 2016;27:37-48.

15. Ye GJ, Budzynski E, Sonnentag P, et al. Safety and biodistribution evaluation in cynomolgus macaques of rAAV2tYF-CB-hRS1, a recombinant adeno-associated virus vector expressing retinoschisin. Hum Gene Ther Clin Dev 2015;26: 165-176.

16. Conway JE, Zolotukhin S, Muzyczka N, et al. Recombinant adeno-associated virus type 2 replication and packaging is entirely supported by a herpes simplex virus type 1 amplicon expressing Rep and Cap. J Virol 1997;71:8780-8789.

17. Conway JE, Rhys CM, Zolotukhin I, et al. Hightiter recombinant adeno-associated virus production utilizing a recombinant herpes simplex virus type I vector expressing AAV-2 Rep and Cap. Gene Ther 1999;6:986-993.

18. Falk DJ, Soustek MS, Todd AG, et al. Comparative impact of AAV and enzyme replacement therapy on respiratory and cardiac function in adult Pompe mice. Mol Ther Methods Clin Dev 2015;2:15007.

19. Potter $M$, Lins $B$, Mietzsch $M$, et al. A simplified purification protocol for recombinant adenoassociated virus vectors. Mol Ther Methods Clin Dev 2014;1:14034.

20. Trybala E, Liljeqvist JA, Svennerholm B, et al. Herpes simplex virus types 1 and 2 differ in their interaction with heparan sulfate. J Virol 2000;74 9106-9114

21. Carrasco L, Otero MJ, Castrillo J. Modification of membrane permeability by animal viruses. Pharmacol Ther 1989;40:171-212.

22. Cecchini S, Virag T, Kotin RM. Reproducible high yields of recombinant adeno-associated virus produced using invertebrate cells in 0.02-to 200liter cultures. Hum Gene Ther 2011;22:10211030.

23. Clark KR. Recent advances in recombinant adenoassociated virus vector production. Kidney Int 2002;61:S9-S15.

24. Buclez P-0, Dias Florencio G, Relizani K, et al Rapid, scalable, and low-cost purification of recombinant adeno-associated virus produced by baculovirus expression vector system. Mol Ther Methods Clin Dev 2016;3:16035.

25. Mietzsch M, Casteleyn V, Weger S, et al. OneBac 2.0: Sf 9 cell lines for production of AAV5 vectors with enhanced infectivity and minimal encapsidation of foreign DNA. Hum Gene Ther 2015; 26:688-697.

26. Flotte TR, Trapnell BC, Humphries M, et al. Phase 2 clinical trial of a recombinant adeno-associated viral vector expressing $\alpha_{1}$-antitrypsin: interim results. Hum Gene Ther 2011;22:1239-1247.

Received for publication October 12, 2016: accepted after revision January 23, 2017.

Published online: January 23, 2017. 